\title{
The quorum sensing regulator OpaR is a repressor of polar flagellum genes in Vibrio parahaemolyticus
}

\author{
Renfei Lu' ${ }^{1}$, Junfang Sun ${ }^{1}$, Yue Qiu ${ }^{3}$, \\ Miaomiao Zhang ${ }^{3}$, Xingfan Xue ${ }^{3}$, Xue Li ${ }^{1}$, \\ Wenhui Yang ${ }^{4}$, Dongsheng Zhou, \\ Lingfei $\mathrm{Hu}^{4 *}$, and Yiquan Zhang ${ }^{2,3 *}$ \\ ${ }^{1}$ Department of Clinical Laboratory, Nantong Third Hospital Affiliated \\ to Nantong University, Nantong 212006, P. R. China \\ ${ }^{2}$ Wuxi School of Medicine, Jiangnan University, Wuxi 214122, \\ P. R. China \\ ${ }^{3}$ School of Medicine, Jiangsu University, Zhenjiang 212013, P. R. China \\ ${ }^{4}$ State Key Laboratory of Pathogen and Biosecurity, Beijing Institute of \\ Microbiology and Epidemiology, Beijing 100071, P. R. China \\ (Received Dec 1, 2020 / Revised Mar 29, 2021 / Accepted Apr 22, 2021)
}

Vibrio parahaemolyticus possesses two types of flagella: a single polar flagellum (Pof) for swimming and the peritrichous lateral flagella (Laf) for swarming. Expression of Laf genes has previously been reported to be regulated by the quorum sensing $(\mathrm{QS})$ regulators $\mathrm{Aph} A$ and $\mathrm{OpaR}$. In the present study, we showed that OpaR, the QS regulator at high cell density (HCD), acted as a negative regulator of swimming motility and the transcription of Pof genes in V. parahaemolyticus. OpaR bound to the promoter-proximal DNA regions of flgAMN, flgMN, and flgBCDEFGHIJ within the Pof gene loci to repress their transcription, whereas it negatively regulates the transcription of $f l g K L-f l a C$ in an indirect manner. Thus, this work investigated how QS regulated the swimming motility via direct action of its master regulator OpaR on the transcription of Pof genes in V. parahaemolyticus.

Keywords: Vibrio parahaemolyticus, polar flagellum, quorum sensing, OpaR

\section{Introduction}

Vibrio parahaemolyticus is a Gram-negative halophilic bacterium that naturally inhabits marine ecosystems. It possesses dual flagellar systems, a single polar flagellum (Pof) for swimming in liquids and the peritrichous lateral flagella (Laf) for swarming over surfaces or in viscous liquids (McCarter, 2004). The Pof is continuously produced, whereas the Laf are expressed only under certain conditions (McCarter and Silverman, 1989; Kawagishi et al., 1996). In addition, unlike that of Pof genes, the expression of Laf genes is highly regulated by numerous transcriptional regulators, including LafK (Gode-

*For correspondence. (Y. Zhang) E-mail: zhangyiquanq@163.com / (L. Hu)

E-mail: huling03@163.com

Copyright (c) 2021, The Microbiological Society of Korea
Potratz et al., 2011), SwrZ (Jaques and McCarter, 2006), SwrT (Jaques and McCarter, 2006), H-NS (Wang et al., 2018), VapH (Park et al., 2005), CalR (Gode-Potratz et al., 2010), and the quorum sensing (QS) regulators AphA and OpaR (Jaques and McCarter, 2006; Wang et al., 2013a; Lu et al., 2019). Vibrio parahaemolyticus swimming motility has been shown to be activated by OxyR (Chung et al., 2016), AphA (Wang et al., 2013a), and ToxR (Chen et al., 2018), but detailed information on its regulatory mechanisms is lacking. Overall, regulation of Pof genes is far from elucidated.

QS is a cell-to-cell communication process used by bacteria to regulate gene expression via detection of signaling molecules called autoinducers, which are produced by bacteria in response to changes in bacterial cell density ( $\mathrm{Ng}$ and Bassler, 2009). QS generally uses downstream master regulators to control gene expression ( $\mathrm{Ng}$ and Bassler, 2009). It has been shown that AphA and OpaR are the two master QS regulators in V. parahaemolyticus, and their highest expression levels occur at low cell density (LCD) and high cell density (HCD), respectively (Sun et al., 2012; Zhang et al., 2012). Both individually and together, they regulate multiple cellular pathways in $V$. parahaemolyticus, including virulence factor production, motility, and biofilm formation (Henke and Bassler, 2004; van Kessel et al., 2013; Wang et al., 2013a, 2013b; Lu et al., 2018, 2019).

The swarming motility regulatory mechanisms by AphA and OpaR have been elucidated in V. parahaemolyticus (Lu et al., 2019), but the swimming motility mechanisms of these two regulators are still lacking. In the present study, we showed that OpaR bound to the promoter-proximal DNA regions of $f l g A M N$, flgMN, and flgBCDEFGHIJ within the Pof gene loci to repress their transcription, whereas it represses flgKLflaC transcription in an indirect manner. Thus, OpaR repressed swimming motility by acting directly on the regulatory regions of Pof genes.

\section{Materials and Methods}

\section{Bacterial strains and growth conditions}

The pandemic $V$. parahaemolyticus strain RIMD 2210633 was used as the wild-type (WT) strain (Makino et al., 2003). Nonpolar $o p a R$ deletion mutant derived from the WT strain, termed $\triangle o p a R$, and the complementary mutant strain $\triangle o p a R /$ pBAD33-opaR, termed C- $\triangle o p a R$, were constructed as described in our previous studies (Zhang et al., 2012, 2019). The empty pBAD33 was also introduced into WT and $\triangle o p a R$ to generate $\mathrm{WT} / \mathrm{pBAD} 33$ and $\triangle o p a R / \mathrm{pBAD} 33$ to counteract the effects of arabinose and chloramphenicol on bacterial growth and physiology (Zhang et al., 2019). 
Glycerol stocks of bacterial cells were inoculated into $5 \mathrm{ml}$ of $2.5 \%(\mathrm{w} / \mathrm{v})$ Bacto heart infusion (HI) broth (BD Biosciences) at $37^{\circ} \mathrm{C}$ with shaking at $200 \mathrm{rpm}$ for $12-14 \mathrm{~h}$. The resulting cell cultures were diluted 50 -fold into $5 \mathrm{ml}$ of fresh $\mathrm{HI}$ broth and then cultured at $37^{\circ} \mathrm{C}$ to reach an optical density at 600 $\mathrm{nm}\left(\mathrm{OD}_{600}\right)$ value of approximately 1.0 to 1.4 . The cell cultures were then diluted 1,000-fold into $10 \mathrm{ml}$ OTN broth (1\% [w/v] Oxoid tryptone [Oxoid] supplemented with $2 \%$ $[\mathrm{w} / \mathrm{v}] \mathrm{NaCl}[\mathrm{Merck}])$ for a third round of incubation and then harvested at the required cell densities. When necessary, the medium was supplemented with $100 \mu \mathrm{g} / \mathrm{ml}$ gentamicin.

\section{Swimming motility assay}

For the swimming motility assay (Wang et al., 2013a), $2 \mu \mathrm{l}$ of cell culture from the second inoculation in $\mathrm{HI}$ broth was inoculated into the semi-solid swim plates (1\% Oxoid tryptone, $2 \% \mathrm{NaCl}$ [Merck], and $0.5 \%$ Difco Noble agar [BD Biosciences]). The diameter of the area covered by the swimming bacteria was measured per hour after incubation at $37^{\circ} \mathrm{C}$.

\section{Quantitative polymerase chain reaction ( $\mathrm{qPCR}$ ) analysis}

Total RNA was extracted using TRIzol Reagent (Invitrogen). The cDNA was generated from $2 \mu \mathrm{g}$ of each RNA sample using the FastKing First Strand cDNA Synthesis Kit (Tiangen Biotech) according to the manufacturer's instructions. The qPCR assay was performed using a LightCycler system (Roche) with SYBR Green master mix (Gao et al., 2011). The relative mRNA levels of each target gene were determined using the $2^{-\Delta \Delta \mathrm{Ct}}$ method (Gao et al., 2011). A mean ratio of two was used as the cutoff value for statistical significance. Experiments were performed independently at least three times. Primers used in the present study are listed in Table 1.

\section{Preparation of $6 \times$ His-tagged OpaR (His-OpaR) protein}

The entire coding region of $o p a R$ was cloned into the pET28a plasmid, and then transformed into Escherichia coli BL21DE3 cells for protein (His-OpaR) expression (Kleber-Janke and Becker, 2000). Expression and purification of His-OpaR was carried out as described previously (Zhang et al., 2012). The purity of the purified His-OpaR was analyzed by $10 \%$ sodium dodecyl sulphate (SDS)-polyacrylamide gel electrophoresis.

\section{Electrophoresis mobility shift assay (EMSA)}

The EMSA was performed as previously described (Zhang et al., 2012, 2017). Briefly, the $5^{\prime}$-ends of the promoter DNA regions of each target gene were labeled with $\left[\gamma^{-}{ }^{32} \mathrm{P}\right]-\mathrm{ATP}$. The EMSA was performed in a $10 \mu \mathrm{l}$ reaction volume containing binding buffer ( $1 \mathrm{mM} \mathrm{MgCl}_{2}, 0.5 \mathrm{mM}$ EDTA, $0.5 \mathrm{mM}$ DTT, $50 \mathrm{mM} \mathrm{NaCl}, 10 \mathrm{mM}$ Tris-HCl; $\mathrm{pH} 7.5$, and $0.05 \mathrm{mg} / \mathrm{ml}$ salmon sperm DNA), labeled DNA probe (100-200 CPM/ $\mu \mathrm{l})$, and increasing amounts of His-OpaR. Two controls were included: (1) cold probe as a specific DNA competitor (unlabeled corresponding promoter DNA fragments) and (2) nonspecific protein competitor (rabbit anti-F1-protein polyclonal antibodies). The EMSA reactions were incubated at room temperature for $20 \mathrm{~min}$, and the binding products were analyzed in native $4 \%(\mathrm{w} / \mathrm{v})$ polyacrylamide gels. The results were detected by autoradiography after exposure to Fuji Medical X-ray film (Fujifilm Corp.).

\section{DNase I footprinting}

For DNase I footprinting (Zhang et al., 2012, 2017), promoter DNA regions with a single ${ }^{32} \mathrm{P}$-labeled end were generated by

Table 1. Oligonucleotide primers used in this study

\begin{tabular}{|c|c|}
\hline Target & Primers (forward/reverse, $5^{\prime}-3^{\prime}$ ) \\
\hline \multicolumn{2}{|l|}{ qPCR } \\
\hline flgM (VP0771) & ATTCAAGTGCGACATCAAG/CGGAGAAGCTGCCATATC \\
\hline flgA (VP0772) & ATTGCCCTGAACCACTCG/ATCGGTACTGAAGAGGAGACAC \\
\hline$f l g B(\mathrm{VP} 0775)$ & ACAAGGCACTAGGCATCC/GACCATCTGTTCGGCTAAG \\
\hline flgK (VP0785) & GCCGTCAGTCAGTGATTC/GTAGAGGACAGGTTGAGTTC \\
\hline \multicolumn{2}{|l|}{ LacZ fusion } \\
\hline $\operatorname{flg} M(\mathrm{VP} 0771)$ & GCGCTCTAGATGTCCCTGTTCGTGTCTC/GCGCGAATTCATGTCGCACTTGAATCGTTAC \\
\hline flgA (VP0772) & GCGCGTCGACTTTCTTGCTCCGCTTGTG/GCGCGAATTCCTGATTATCTGTTGCCGAATGC \\
\hline$f \lg B(\mathrm{VP} 0775)$ & GCGCGTCGACTTAGGTCGTGGTCTTTCG/GCGCGAATTCTTGCCGCTTGTAACTCCTTG \\
\hline flgK (VP0785) & GCGCGTCGACAACCATCATTGCTTCTTG/GCGCGAATTCTGCTTGTAGAGGACAGGTTGAG \\
\hline \multicolumn{2}{|l|}{ Protein expression } \\
\hline opaR & AGCGGGATCCATGGACTCAATTGCAAAGAG/AGCGAAGCTTTTAGTGTTCGCGATTGTAG \\
\hline \multicolumn{2}{|l|}{ EMSA } \\
\hline flgM (VP0771) & CGTAGCGTTCGTCTTGGTG/GCCATGTTTAACCTTTGCCTTG \\
\hline flgA (VP0772) & TTGCCAGGCTCAAATCAATTAC/AATGCCGATAGACTTAGCGTAC \\
\hline$f \lg B(\mathrm{VP} 0775)$ & AATCCAGGTATCATTTACAAGC/TGGATGCCTAGTGCCTTGTC \\
\hline flgK (VP0785) & AACCATCATTGCTTCTTG/TGCTTGTAGAGGACAGGTTGAG \\
\hline \multicolumn{2}{|c|}{ DNase I footprinting } \\
\hline flgM (VP0771) & CGTAGCGTTCGTCTTGGTG/TTCCGCAATGTCAGTCACTATG \\
\hline flgA (VP0772) & VTTGCCAGGCTCAAATCAATTAC/TGACGGGGATTCTTGACTCG \\
\hline$f \lg B(\mathrm{VP} 0775)$ & GCTAATCTGTTGTCGGACTTTG/TGGATGCCTAGTGCCTTGTC \\
\hline flgK (VP0785) & TTGCAGCAACGTGGAGATTC/TGAGCAGTAAGCACACTTTGAG \\
\hline
\end{tabular}


PCR with either sense or antisense primers, end-labeled, and purified using QiaQuick columns (Qiagen). DNA binding was performed in a $10 \mu \mathrm{l}$ reaction volume containing binding buffer (the same used for EMSA), labeled DNA fragment (2-5 pmol), and increasing amounts of His-OpaR. The reactions were incubated at room temperature for $30 \mathrm{~min}$. Prior to digestion, $10 \mu \mathrm{l}$ of a $\mathrm{Ca}^{2+} / \mathrm{Mg}^{2+}$ solution $\left(5 \mathrm{mM} \mathrm{CaCl}_{2}\right.$ and $10 \mathrm{mM} \mathrm{MgCl}_{2}$ ) was added to each reaction and incubated for $1 \mathrm{~min}$ at room temperature. Optimized RQ1 RNase-Free DNase I (Promega) was added to each reaction mixture and then incubated at room temperature for $40-90 \mathrm{sec}$. The reaction was quenched by adding $9 \mu \mathrm{l}$ of stop solution $(200 \mathrm{mM}$ $\mathrm{NaCl}, 30 \mathrm{mM}$ EDTA, and 1\% SDS), followed by incubation for $1 \mathrm{~min}$ at room temperature. The partially digested DNA samples were extracted with phenol/chloroform, precipitated with ethanol, and analyzed on $6 \%$ polyacrylamide/8 $\mathrm{M}$ urea gels. Protected regions were identified by comparison with the sequence ladder. The templates for Sanger sequencing were the same as the DNA fragments used in the DNase I footprinting assay. Sanger sequencing was carried out using an AccuPower \& Top DNA Sequencing Kit (Bioneer) according to the manufacturer's instructions. Radioactive species were detected by autoradiography after exposure to Fuji Medical X-ray film (Fujifilm Corp.).

\section{Statistical methods}

Swimming motility and qPCR assays were carried out with at least three independent bacterial cultures, and the values were expressed as the mean \pm standard deviation (SD). Paired Student's $t$-tests were used to calculate statistically significant differences. $P<0.01$ was considered statistically significant. Data for EMSA and DNase I footprinting assays were collected from at least two independent biological replicates.

\section{Results}

\section{OpaR represses swimming motility}

Regulation of swimming motility by the QS regulator OpaR was investigated, and the results showed that swimming capacity was significantly increased in $\triangle o p a R$ compared with

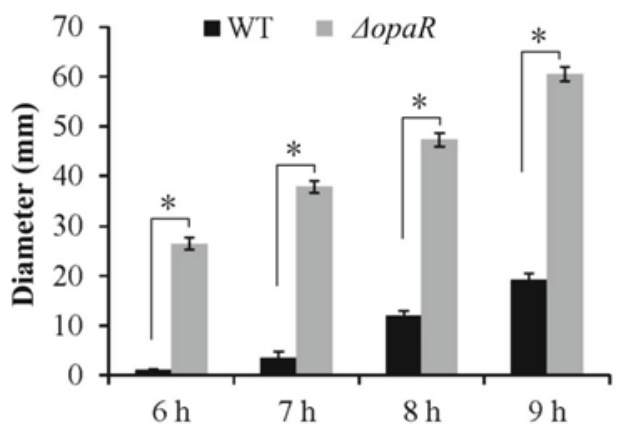

Fig. 1. Regulation of swimming motility by OpaR. Swimming was evaluated by measuring the diameter of the area covered by swimming bacterial cells in semi-solid swimming plates. Results are expressed as the mean \pm SD and were analyzed by paired Student's $t$-test. The asterisk $\left.{ }^{*}\right)$ represents $P$ $<0.01$.

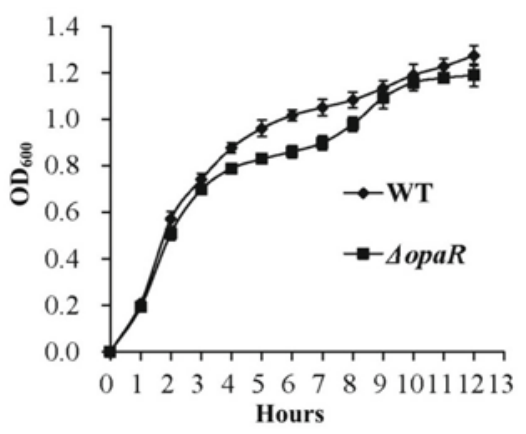

Fig. 2. Growth curves of WT and $\triangle o p a R$. The WT and $\triangle o p a R$ strains were grown in the OTN broth at $37^{\circ} \mathrm{C}$ with shaking at $200 \mathrm{rpm}$, and the $\mathrm{OD}_{600}$ values of each strain were monitored at 1-h intervals. Experiments were performed at least two times with three replicates per trial for each strain.

WT (Fig. 1) at all time points tested. In addition, the diameter of the area covered by swimming bacterial cells increased with cultivation time for both the WT and $\triangle o p a R$ strains. These observations suggested that OpaR was a repressor of swimming motility in $V$. parahaemolyticus.

\section{Growth of WT and $\triangle o p a R$ strains}

The WT and $\triangle o p a R$ strains were grown in OTN broth at $37^{\circ} \mathrm{C}$ with shaking at $200 \mathrm{rpm}$, and $\mathrm{OD}_{600}$ values of each strain were monitored at $1 \mathrm{~h}$ intervals to create growth curves. As shown in Fig. 2, indistinguishable growth rates were ob-
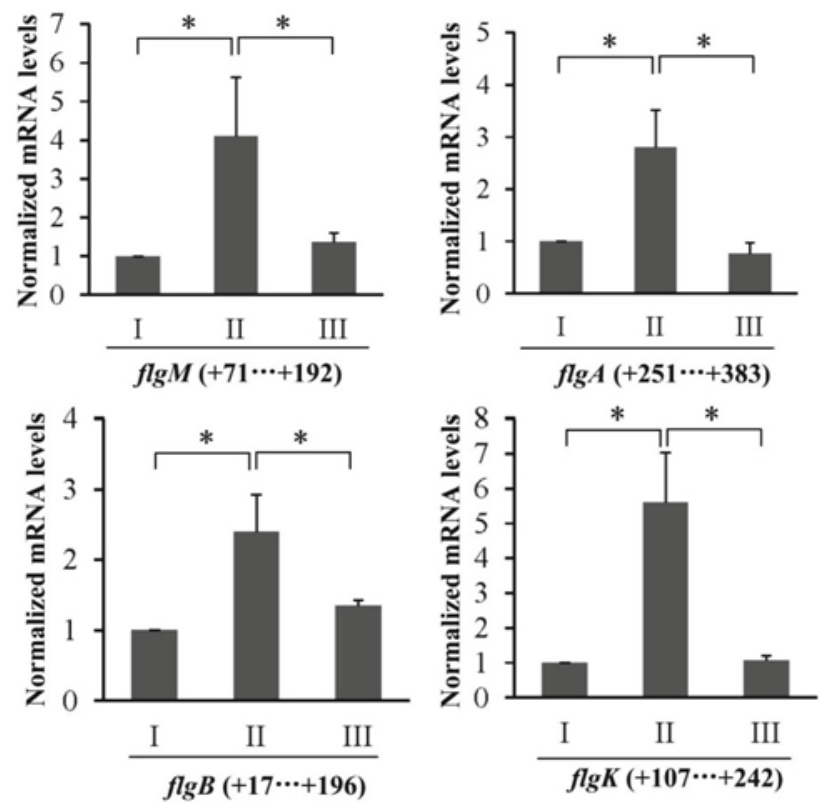

Fig. 3. Negative regulation of Pof genes by OpaR. Negative and positive numbers represent the nucleotide positions upstream and downstream of each target gene. The relative mRNA levels of each target gene were compared between WT/pBAD33, $\Delta o p a R / p B A D 33$, and C- $\Delta o p a R$. I, II, and III represent $\mathrm{WT} / \mathrm{pBAD} 33, \Delta o p a R / \mathrm{pBAD} 33$, and C- $\Delta o p a R$, respectively. The asterisk $\left(^{*}\right)$ represents $P<0.01$, which was determined by a paired Student's $t$-test. 
served for these two strains in the medium, suggesting that deletion of opaR did not affect the growth of $V$. parahaemolyticus in OTN broth. Bacterial cells were harvested at the early exponential stage (an $\mathrm{OD}_{600}$ value of approximately 0.8 ) for the following biochemical assays.

\section{Selection of target genes}

The Pof gene system comprises approximately 60 genes, and most of these genes are located in two regions, containind at least 11 operons: flgAMN, flgMN, cheVR, flgBCDEFGHIJ, flgKL-flaC, flgKL, flaDE, flaAGHIJK, flaKLM, fliEFGHIJKLMNOPQR-flhB, and flhAFG-fliA-cheYZAB-ORF1-ORF2cheW-ORF3 (Kim and McCarter, 2000; McCarter, 2001).
The $500 \mathrm{bp}$ DNA regions upstream of the first genes in these operons were downloaded from the WT genome. The DNAbinding box of OpaR (Zhang et al., 2012) was used to predict the presence of OpaR box-like sequences within the DNA regions using the matrix scan tool (van Helden, 2003). At a cutoff value score of 6 (Zhang et al., 2012), no OpaR box-like sequences were detected for any DNA sequence tested (data not shown).

The OpaR box has been proven to be useful for the prediction of direct OpaR targets, but because it was created from LuxR homolog binding sites, using this box may lead to potential binding sites being assigned lower scores and some potential OpaR sites to be missed (Zhang et al., 2012; Kernell Burke et al., 2015). Thus, the first genes in the flgAMN, flgMN,

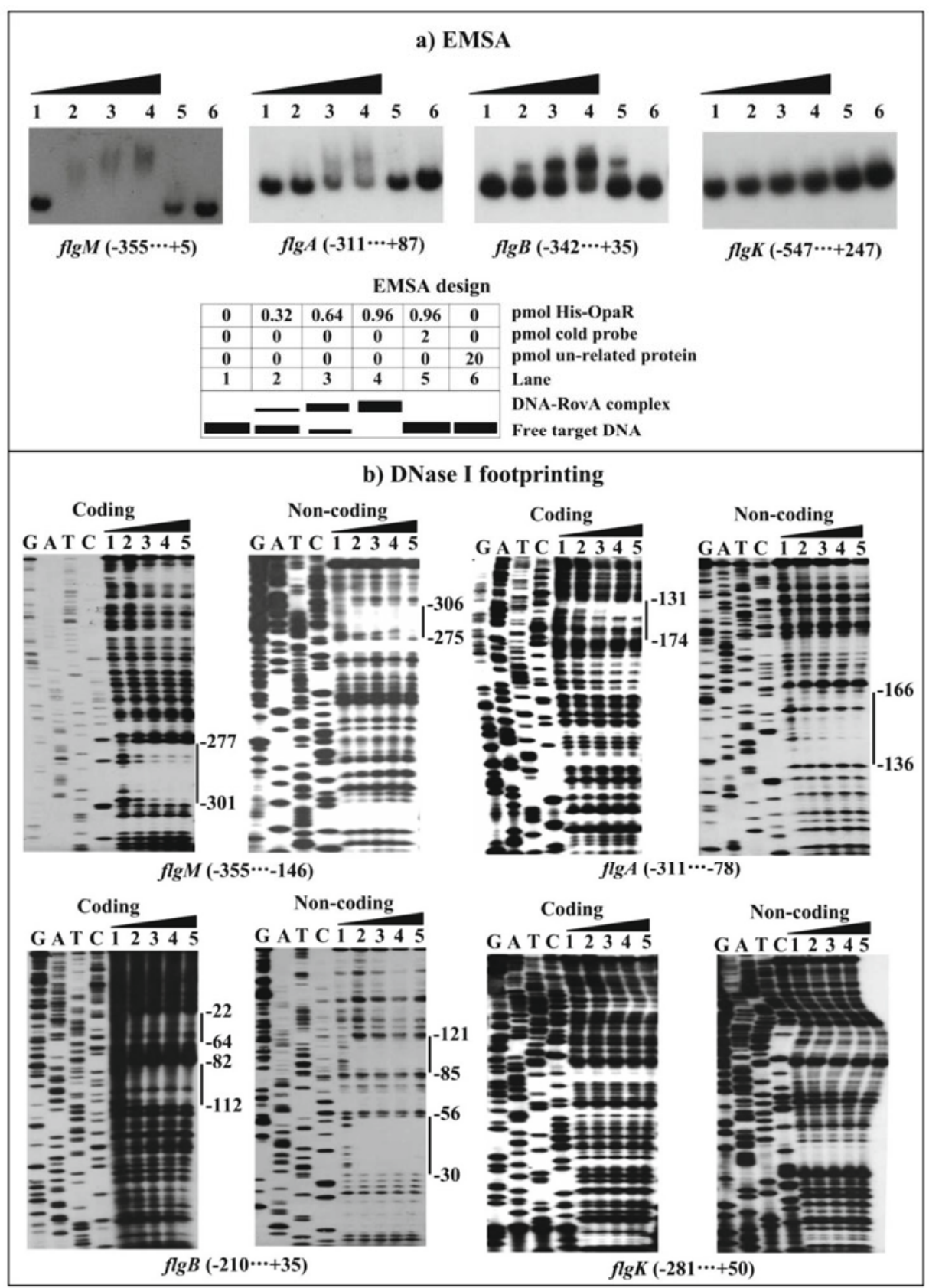

Fig. 4. Binding of His-OpaR to the promoter DNA regions of Pof genes. Negative and positive numbers represent the nucleotide positions upstream and downstream of each target gene. (A) EMSA. The entire promoter DNA region of each target gene was incubated with increasing amounts of His-OpaR and then subjected to $6 \%(\mathrm{w} / \mathrm{v})$ polyacrylamide gel electrophoresis. The EMSA design is shown below. (B) DNase I footprinting. Lanes G, $\mathrm{A}, \mathrm{T}$, and $\mathrm{C}$ represent Sanger sequencing reactions. Labeled coding or non-coding DNA probes were incubated with increasing amounts of purified His-OpaR (Lanes $1,2,3,4$, and 5 contained $0,3,6,9$, and 12 pmol, respectively), and were subjected to DNase I footprinting. The protected regions are indicated with vertical bars. 
flgBCDEFGHIJ, and flgKL-flaC operons were randomly selected as target genes for subsequent gene regulation studies.

\section{OpaR represses the transcription of Pof genes}

The qPCR assays were employed to detect differences in mRNA levels of Pof genes between WT/pBAD33, $\Delta o p a R /$ pBAD33, and C- $\triangle o p a R$. As shown in Fig. 3, the mRNA level of each target gene was significantly increased in $\Delta o p a R /$ pBAD33 relative to $\mathrm{WT} / \mathrm{pBAD} 33$ and $\mathrm{C}-\triangle o p a R$, whereas expression levels of each target gene were restored in C- $\Delta o p a R$. These results suggested that OpaR repressed Pof gene transcription in $V$. parahaemolyticus.

\section{Direct binding of His-OpaR to the regulatory DNA regions of Pof genes}

The promoter-proximal DNA region of each target gene was amplified and subjected to EMSA with purified His-OpaR. As shown in Fig. 4A, His-OpaR was able to bind to the promoter-proximal DNA regions of $f \lg M, f \lg A$, and $f i g B$ in a dose-dependent manner, but it was unable to bind to the promoter-proximal DNA region of $f l g K$ at all protein amounts tested. As further determined by DNase I footprinting (Fig. $4 \mathrm{~B})$, His-OpaR protected single DNA regions located from 306 to $275 \mathrm{bp}$ and from 174 to $131 \mathrm{bp}$ within the upstream DNA regions of flgM and $f l g A$, respectively, against DNase I digestion, whereas it protected two different DNA regions from 121 to $82 \mathrm{bp}$ and 64 to $22 \mathrm{bp}$ upstream of $f \mathrm{lg} B$. These protected regions were considered OpaR binding sites. However, no His-OpaR protected DNA regions were detected in the upstream DNA region of $f l g K$. Thus, OpaR directly repressed the transcription of $f \lg M$, $f \lg A$, and $f \lg B$, whereas it indirectly repressed $f \lg K$ transcription.

\section{Discussion}

Vibrio parahaemolyticus swimming motility, powered by the Pof, is a mode of movement of individual bacterial cells in liquid environments (McCarter, 2004). In contrast, swarming motility, powered by the Laf, is a process by which bacteria move on surfaces or in viscous liquids in a coordinated manner (McCarter, 2004). More studies have focused on the Laf by far, especially those that involve the investigation of

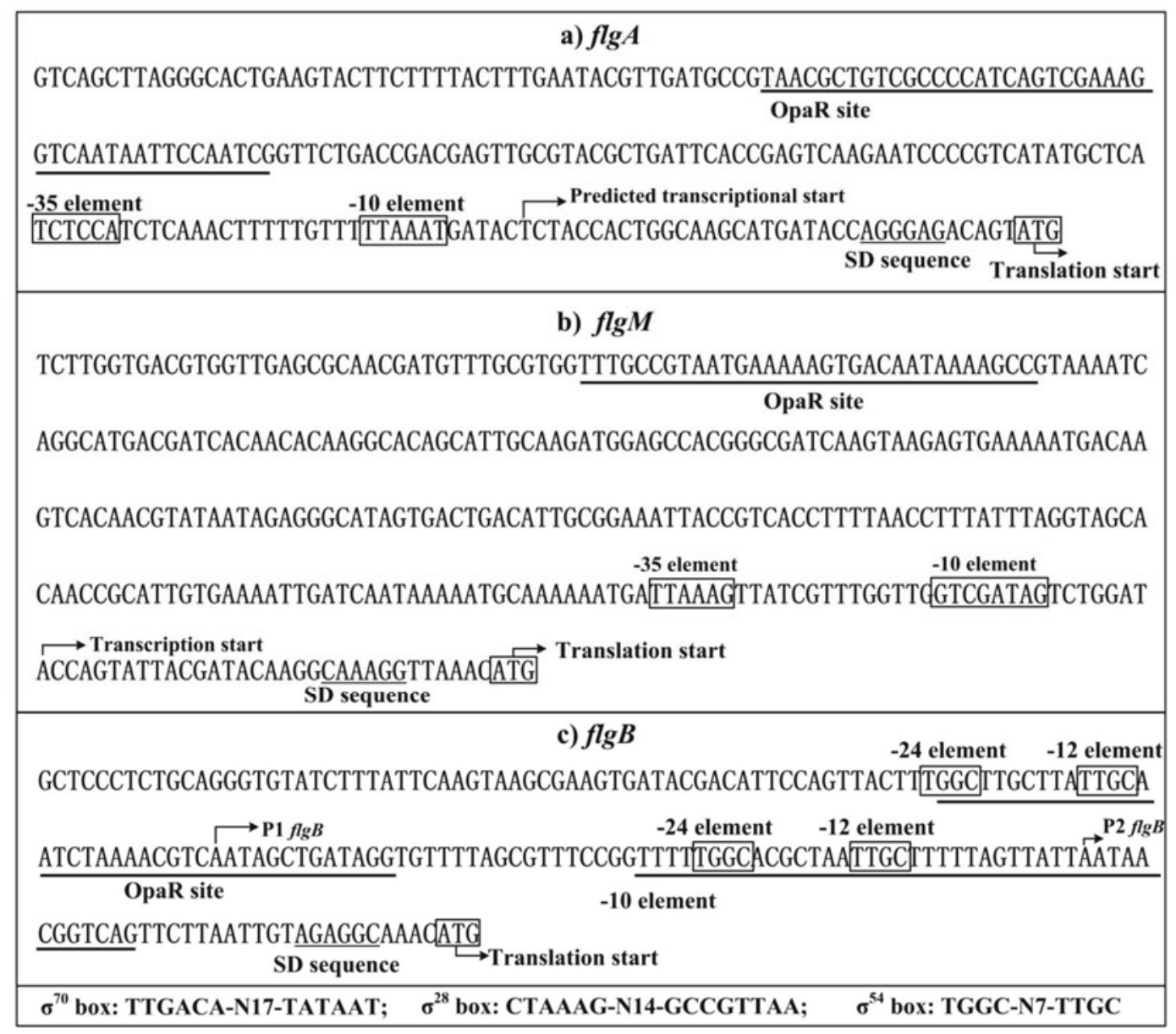

Fig. 5. Organization of promoter DNA regions of Pof genes. The promoter DNA regions of selected target genes were derived from V. parahaemolyticus RIMD 221063. The translation and transcription start sites are shown with bent arrows. The predicted core promoters ( -10 and -35 or -12 and -24 elements) are boxed. The OpaR binding sites and the SD sequences are underlined. The $\sigma^{70}, \sigma^{28}$, and $\sigma^{54}$ boxes are shown at the bottom of the Figure. 
its roles in biofilm formation and the regulation of its coding genes (McCarter and Silverman, 1989; McCarter, 2004; Park et al., 2005; Jaques and McCarter, 2006; Gode-Potratz et al., 2010; Wang et al., 2013a, 2018; Chung et al., 2016; Lu et al., 2019). However, more than 50 gene products are involved in assembling a functional Pof, and thus, bacteria must also employ complex regulatory systems to control Pof gene expression (McCarter, 2001). AphA, the QS regulator at LCD, plays a positive role in regulating swimming in $V$. parahaemolyticus, and deletion of aphA significantly decreases swimming motility capacity relative to WT (Wang et al., 2013a). ToxR also acts as an activator of swimming motility in $V$. parahaemolyticus, and the swimming motility capacity of the $\Delta$ toxR strain is dramatically decreased relative to WT (Chen et al., 2018). Swimming capacity is also significantly inhibited in the $\triangle o x y R$ strain relative to $\mathrm{WT}$, suggesting that $\mathrm{OxyR}$ is also a positive regulator of swimming motility in $V$. parahaemolyticus (Chung et al., 2016). In addition, the VcrD1 protein, a component of the $V$. parahaemolyticus type III secretion system 1, plays a key role in flagellar morphogenesis via regulation of the expression and secretion of flagellar components (Noh et al., 2015).

In the present work, we showed that OpaR, the QS regulator at HCD, acted as a negative regulator of swimming motility and Pof gene transcription in V. parahaemolyticus. OpaR bound to the promoter-proximal DNA regions of flgAMN, $f \lg M N$, and $f l g B C D E F G H I J$ within the Pof gene loci to repress their transcription, whereas it negatively regulated the transcription of flgKL-flaC in an indirect manner. Thus, the repression of swimming motility by OpaR in $V$. parahaemolyticus occurred via the direct and negative regulatory actions of OpaR on the transcription of Pof genes. OpaR homologs in other Vibrio species, including SmcR, VtpR, HapR, and LuxR, have been demonstrated to be involved in regulating Pof biosynthesis (Nielsen et al., 2006; Hasegawa and Hase, 2009; Kim et al., 2012; Yang and Defoirdt, 2015). In V. vulnificus and $V$. tubiashii, SmcR and VtpR repress flagellar biosynthesis and swimming motility (Hasegawa and Hase, 2009; Kim et al., 2012), whereas LuxR and HapR activate the expression of flagellar genes and swimming motility in $V$. harveyi and V. cholerae (Nielsen et al., 2006; Yang and Defoirdt, 2015). Thus, regulation of flagellar genes by the master QS regulator at HCD may be dependent on genetic background.

Many reports have demonstrated that the two master QS regulators AphA and OpaR coordinately and inversely regulate the transcription of their target genes in $V$. parahaemolyticus RIMD2210633 during the transition from LCD to HCD (Sun et al., 2012; Zhang et al., 2012, 2019; Wang et al., 2013b; Zhou et al., 2013; Lu et al., 2019). Transcriptional regulation of Pof genes by AphA and OpaR is another example of this phenomenon. The motility mediated by the Pof is closely related to biofilm formation and virulence (Enos-Berlage et al., 2005; Yildiz and Visick, 2009; Yang and Defoirdt, 2015). Interestingly, it has been suggested that the Pof is involved in the initial stage of biofilm formation, but its biosynthesis is repressed after the biofilm is formed (Yildiz and Visick, 2009; Zhu et al., 2013). OpaR activate the expression of biofilm-associated genes at HCD (McCarter, 1998; Enos-Berlage and McCarter, 2000). The possible benefits of OpaR-mediated repression of Pof gene expression are not yet clear. Down- regulation of Pof genes by the master QS regulator OpaR at HCD suggests that swimming motility is not as important during the late growing period or during the later stage of infection. Inhibiting the expression of Pof genes could help bacteria conserve limited nutrients.

The promoter organization of $f l g A M N$, flgMN, and $f l g B C D$ $E F G H I J$ were reconstructed herein by collecting data on the translation/transcription start sites (Kim and McCarter, 2000; McCarter, 2001), core promoter elements (-10/-35 and -12/ -24), OpaR binding sites, and Shine-Dalgarno (SD) sequences (Fig. 5). The flgMN operon is part of the larger flgAMN operon, that is, the flgMN genes can also be transcribed from the flgAMN promoter (Kim and McCarter, 2000; McCarter, 2001). A $\sigma^{28}$-dependent promoter was previously identified for the initiation of flgMN (also seen in Fig. 5B) (Kim and McCarter, 2000), but no transcription start sites have been determined for flgAMN. However, a $\sigma^{70}$-dependent promoter was predicted for flgAMN using the online Softberry tool (http://linux1.softberry.com/berry.phtml) (Fig. 5A). The OpaR binding site for flgAMN or flgMN was located far upstream of the -35 element, which was an unusual position for a regulator that repressed its target genes. There may be unknown regulators that activated the transcription of flgAMN and $f l g M N$, and the binding of OpaR may interfere with their functions. Two $\sigma^{54}$-dependent promoters, named P1 and P2, have been identified upstream of $f l g B C D E F G H I J$ that initiate its transcription (Fig. 5C) (Kim and McCarter, 2000). The two OpaR binding sites for flgBCDEFGHIJ entirely overlapped each of the promoters (Fig. $5 \mathrm{C}$ ). Thus, OpaR repressed the transcription of flgBCDEFGHIJ, possibly via direct interference with RNA polymerase. In addition, the binding of OpaR to these two sites may lead to the occurrence of hairpins, thereby causing interference with the production of mRNAs.

\section{Acknowledgements}

This work was supported by the Postdoctoral Science Foundation Funded Project of Jiangsu Province (Grant No. 2020Z102), the National Natural Science Foundation of China (82072239) and the China Postdoctoral Science Foundation (Grant No. 2020M681513).

\section{Conflict of Interest}

The authors have no conflicts of interest to report.

\section{References}

Chen, L., Qiu, Y., Tang, H., Hu, L.F., Yang, W.H., Zhu, X.J., Huang, X.X., Wang, T., and Zhang, Y.Q. 2018. ToxR is required for biofilm formation and motility of Vibrio parahaemolyticus. Biomed. Environ. Sci. 31, 848-850.

Chung, C.H., Fen, S., Yu, S.C., and Wong, H. 2016. Influence of $o x y R$ on growth, biofilm formation, and mobility of Vibrio parahaemolyticus. Appl. Environ. Microbiol. 82, 788-796.

Enos-Berlage, J.L., Guvener, Z.T., Keenan, C.E., and McCarter, L.L. 2005. Genetic determinants of biofilm development of opaque and translucent Vibrio parahaemolyticus. Mol. Microbiol. 55, 1160- 
1182.

Enos-Berlage, J.L. and McCarter, L.L. 2000. Relation of capsular polysaccharide production and colonial cell organization to colony morphology in Vibrio parahaemolyticus. J. Bacteriol. 182, 55135520 .

Gao, H., Zhang, Y., Yang, L., Liu, X., Guo, Z., Tan, Y., Han, Y., Huang, X., Zhou, D., and Yang, R. 2011. Regulatory effects of cAMP receptor protein (CRP) on porin genes and its own gene in Yersinia pestis. BMC Microbiol. 11, 40.

Gode-Potratz, C.J., Chodur, D.M., and McCarter, L.L. 2010. Calcium and iron regulate swarming and type III secretion in Vibrio parahaemolyticus. J. Bacteriol. 192, 6025-6038.

Gode-Potratz, C.J., Kustusch, R.J., Breheny, P.J., Weiss, D.S., and McCarter, L.L. 2011. Surface sensing in Vibrio parahaemolyticus triggers a programme of gene expression that promotes colonization and virulence. Mol. Microbiol. 79, 240-263.

Hasegawa, H. and Häse, C.C. 2009. TetR-type transcriptional regulator VtpR functions as a global regulator in Vibrio tubiashii. Appl. Environ. Microbiol. 75, 7602-7609.

Henke, J.M. and Bassler, B.L. 2004. Quorum sensing regulates type III secretion in Vibrio harveyi and Vibrio parahaemolyticus. J. Bacteriol. 186, 3794-3805.

Jaques, S. and McCarter, L.L. 2006. Three new regulators of swarming in Vibrio parahaemolyticus. J. Bacteriol. 188, 2625-2635.

Kawagishi, I., Imagawa, M., Imae, Y., McCarter, L., and Homma, M. 1996. The sodium-driven polar flagellar motor of marine Vibrio as the mechanosensor that regulates lateral flagellar expression. Mol. Microbiol. 20, 693-699.

Kernell Burke, A., Guthrie, L.T.C., Modise, T., Cormier, G., Jensen, R.V., McCarter, L.L., and Stevens, A.M. 2015. OpaR controls a network of downstream transcription factors in Vibrio parahaemolyticus BB22OP. PLoS ONE 10, e0121863.

Kim, S.M., Lee, D.H., and Choi, S.H. 2012. Evidence that the Vibrio vulnificus flagellar regulator FlhF is regulated by a quorum sensing master regulator SmcR. Microbiology 158, 2017-2025.

Kim, Y.K. and McCarter, L.L. 2000. Analysis of the polar flagellar gene system of Vibrio parahaemolyticus. J. Bacteriol. 182, 36933704 .

Kleber-Janke, T. and Becker, W.M. 2000. Use of modified BL21(DE3) Escherichia coli cells for high-level expression of recombinant peanut allergens affected by poor codon usage. Protein Expr. Purif. 19, 419-424.

Lu, R., Osei-Adjei, G., Huang, X., and Zhang, Y. 2018. Role and regulation of the orphan AphA protein of quorum sensing in pathogenic Vibrios. Future Microbiol. 13, 383-391.

Lu, R., Tang, H., Qiu, Y., Yang, W., Yang, H., Zhou, D., Huang, X., Hu, L., and Zhang, Y. 2019. Quorum sensing regulates the transcription of lateral flagellar genes in Vibrio parahaemolyticus. Future Microbiol. 14, 1043-1053.

Makino, K., Oshima, K., Kurokawa, K., Yokoyama, K., Uda, T., Tagomori, K., Iijima, Y., Najima, M., Nakano, M., Yamashita, A., et al. 2003. Genome sequence of Vibrio parahaemolyticus: a pathogenic mechanism distinct from that of $V$. cholerae. Lancet 361, 743-749.

McCarter, L.L. 1998. OpaR, a homolog of Vibrio harveyi LuxR, controls opacity of Vibrio parahaemolyticus. J. Bacteriol. 180, 31663173.

McCarter, L.L. 2001. Polar flagellar motility of the Vibrionaceae. Microbiol. Mol. Biol. Rev. 65, 445-462.

McCarter, L.L. 2004. Dual flagellar systems enable motility under different circumstances. J. Mol. Microbiol. Biotechnol. 7, 18-29.

McCarter, L. and Silverman, M. 1989. Iron regulation of swarmer cell differentiation of Vibrio parahaemolyticus. J. Bacteriol. 171,
731-736.

Ng, W.L. and Bassler, B.L. 2009. Bacterial quorum-sensing network architectures. Annu. Rev. Genet. 43, 197-222.

Nielsen, A.T., Dolganov, N.A., Otto, G., Miller, M.C., Wu, C.Y., and Schoolnik, G.K. 2006. RpoS controls the Vibrio cholerae mucosal escape response. PLoS Pathog. 2, e109.

Noh, H.J., Nagami, S., Kim, M.J., Kim, J., Lee, N.K., Lee, K.H., and Park, S.J. 2015. Role of VcrD1 protein in expression and secretion of flagellar components in Vibrio parahaemolyticus. Arch. Microbiol. 197, 397-410.

Park, K.S., Arita, M., Iida, T., and Honda, T. 2005. $v p a H$, a gene encoding a novel histone-like nucleoid structure-like protein that was possibly horizontally acquired, regulates the biogenesis of lateral flagella in trh-positive Vibrio parahaemolyticus TH3996. Infect. Immun. 73, 5754-5761.

Sun, F., Zhang, Y., Wang, L., Yan, X., Tan, Y., Guo, Z., Qiu, J., Yang, R., Xia, P., and Zhou, D. 2012. Molecular characterization of direct target genes and cis-acting consensus recognized by quorumsensing regulator AphA in Vibrio parahaemolyticus. PLoS ONE 7, e44210.

van Helden, J. 2003. Regulatory sequence analysis tools. Nucleic Acids Res. 31, 3593-3596.

van Kessel, J.C., Rutherford, S.T., Shao, Y., Utria, A.F., and Bassler, B.L. 2013. Individual and combined roles of the master regulators AphA and LuxR in control of the Vibrio harveyi quorum-sensing regulon. J. Bacteriol. 195, 436-443.

Wang, L., Ling, Y., Jiang, H., Qiu, Y., Qiu, J., Chen, H., Yang, R., and Zhou, D. 2013a. AphA is required for biofilm formation, motility, and virulence in pandemic Vibrio parahaemolyticus. Int. J. Food Microbiol. 160, 245-251.

Wang, Y., Zhang, Y., Yin, Z., Wang, J., Zhu, Y., Peng, H., Zhou, D., Qi, Z., and Yang, W. 2018. H-NS represses transcription of the flagellin gene lafA of lateral flagella in Vibrio parahaemolyticus. Can. J. Microbiol. 64, 69-74.

Wang, L., Zhou, D., Mao, P., Zhang, Y., Hou, J., Hu, Y., Li, J., Hou, S., Yang, R., Wang, R., et al. 2013b. Cell density- and quorum sensing-dependent expression of type VI secretion system 2 in Vibrio parahaemolyticus. PLoS ONE 8, e73363.

Yang, Q. and Defoirdt, T. 2015. Quorum sensing positively regulates flagellar motility in pathogenic Vibrio harveyi. Environ. Microbiol. 17, 960-968.

Yildiz, F.H. and Visick, K.L. 2009. Vibrio biofilms: so much the same yet so different. Trends Microbiol. 17, 109-118.

Zhang, Y., Gao, H., Osei-Adjei, G., Zhang, Y., Yang, W., Yang, H., Yin, Z., Huang, X., and Zhou, D. 2017. Transcriptional regulation of the type VI secretion system 1 genes by quorum sensing and ToxR in Vibrio parahaemolyticus. Front. Microbiol. 8, 2005.

Zhang, Y., Hu, L., Qiu, Y., Osei-Adjei, G., Tang, H., Zhang, Y., Zhang, R., Sheng, X., Xu, S., Yang, W., et al. 2019. QsvR integrates into quorum sensing circuit to control Vibrio parahaemolyticus virulence. Environ. Microbiol. 21, 1054-1067.

Zhang, Y., Qiu, Y., Tan, Y., Guo, Z, Yang, R, and Zhou, D. 2012. Transcriptional regulation of opaR, qrr2-4 and aphA by the master quorum-sensing regulator OpaR in Vibrio parahaemolyticus. PLoS ONE 7, e34622.

Zhou, D., Yan, X., Qu, F., Wang, L., Zhang, Y., Hou, J., Hu, Y., Li, J., Xin, S., Qiu, J., et al. 2013. Quorum sensing modulates transcription of $c p s Q-m p p A B C$ and $m f p A B C$ in Vibrio parahaemolyticus. Int. J. Food Microbiol. 166, 458-463.

Zhu, S., Kojima, S., and Homma, M. 2013. Structure, gene regulation and environmental response of flagella in Vibrio. Front. Microbiol. 4, 410 . 